\title{
Repensando la programación como formación práctica en Ingeniería: Un estudio de caso en primer año
}

\author{
Rethinking Engineering practical training: a case study on \\ programming in first year of college \\ Natalia Monjelat ${ }^{1 *} \quad$ Guillermo Rodríguez ${ }^{2}$ \\ Recibido 9 de noviembre de 2016, aceptado 3 de enero de 2017 \\ Received: November 9, 2016 Accepted: January 3, 2017
}

\begin{abstract}
RESUMEN
En el presente trabajo se analiza una experiencia llevada adelante en el marco de la actividad curricular "Introducción a la Ingeniería Mecánica" de la Facultad de Ciencias Exactas, Ingeniería y Agrimensura de la Universidad Nacional de Rosario, Argentina. Dicha asignatura integra una modalidad pedagógica de taller, enfatizando la formación práctica en el marco de las actuales políticas latinoamericanas de fortalecimiento de la formación de ingenieros. En este contexto, se propuso la realización de un proyecto final integrador con el objetivo de introducir al alumnado en la resolución de problemas abiertos de ingeniería, proponiendo como consigna la simulación de un sistema mecánico sencillo en un entorno de programación por bloques. Desde un estudio de caso exploratorio con metodología mixta, el artículo expone un análisis de esta experiencia piloto, indagando en las formas en que los participantes se aproximaron al ejercicio propuesto e identificando problemáticas derivadas de la construcción de la simulación. Los resultados mostraron el logro de los objetivos planteados en el taller y evidenciaron el potencial que posee la programación para el fortalecimiento de habilidades vinculadas a la enseñanza práctica. Por otra parte, la mejora de otros aspectos como el trabajo en grupo unido al autoaprendizaje, introducen cambios iniciales en los hábitos de los ingresantes para la resolución de problemas. En síntesis, la experiencia aporta evidencias en torno al potencial de la modalidad de taller para incluir tecnologías dentro de la formación práctica de futuros ingenieros, permitiendo el desarrollo de diferentes habilidades destacadas por las políticas universitarias vigentes.
\end{abstract}

Palabras clave: enseñanza en ingeniería, formación práctica, programación informática, estudio de caso, taller.

\section{ABSTRACT}

This paper presents an experience carried out within the curricular activity "Introduction to Mechanical Engineering" of the Faculty of Sciences, Engineering and Statistics of the National University of Rosario, Argentina. This subject propose a workshop as pedagogical framework, emphasizing practical training in the context of current Latin American policies which aim to strength engineering education. In this context, the realization of an integrative final project was proposed, with the aim to introduce students to the resolution of open engineering problems, simulating a simple mechanical system in a block programming environment. From an exploratory case study with mixed methodology, the article presents

\footnotetext{
1 Instituto Rosario de Investigaciones en Ciencias de la Educación-IRICE (CONICET-UNR). Avenida 27 de Febrero, 210 bis. Rosario, Santa Fe, Argentina. E-mail: monjelat@irice-conicet.gov.ar

2 Facultad de Ciencias Exactas, Ingeniería y Agrimensura. Universidad Nacional de Rosario. Pellegrini 250. Rosario, Santa Fe, Argentina. E-mail: guille@fceia.unr.edu.ar

* Autor de correspondencia
} 
an analysis of this pilot experience, delving into the ways in which participants approached the proposed problem and identifying issues arising from the construction of the simulation. The results showed the achievement of the workshop goals and the potential of programming to strengthen the skills linked to practical teaching. On the other hand, the improvement of other aspects such as group work, together with self-learning, introduce initial changes in students' problem-solving habits. In summary, the experience provides evidence on the potential of the workshop modality to include technologies within the practical training of future engineers, allowing the development of different skills highlighted by current university policies.

Keywords: engineering education, practical training, programming, case study, workshop.

\section{INTRODUCCIÓN}

La enseñanza de la ingeniería se ha convertido en una de las áreas prioritarias de la educación superior durante los últimos años. En este sentido, varios países de América latina, han generado programas para la mejora en la formación de ingenieros. Dichos planes incluyen desde la capacitación docente, pasando por la ampliación en el equipamiento e infraestructura de las facultades, hasta el ordenamiento y renovación de las propuestas académicas de los planes de estudio. Como resultado de estas iniciativas, la Facultad de Ciencias Exactas, Ingeniería y Agrimensura [1] de la Universidad Nacional de Rosario (Argentina) [2], emprendió entre otros compromisos, una reforma curricular integral durante los años 2012 y 2013. Fruto de este trabajo se pusieron en marcha en el año 2014 los nuevos diseños curriculares de las seis carreras de grado de ingeniería que allí se dictan. Particularmente, la carrera de Ingeniería Mecánica en el marco de sus planes de mejora aprobados luego de la Acreditación 2011 realizadas en el marco de la Comisión Nacional de Evaluación y Acreditación Universitaria [3] y del sistema de Acreditación Regional de Carreras Universitarias y la Red de Agencias Nacionales de Acreditación [4] no solo buscó la actualización de contenidos, sino que decidió potenciar las actividades de formación práctica para el desarrollo de habilidades vinculadas a la profesión.

Una de las habilidades de formación práctica que figuran en las resoluciones de organismos nacionales e internacionales en relación a los nuevos perfiles para los egresados, es el uso de las Tecnologías de la Información y Comunicación (TIC) necesarias para el desempeño profesional. Actualmente y en el marco de la sociedad del conocimiento, los ingenieros deben utilizar diversos entornos de manera autónoma y desarrollar nuevas utilidades de los mismos. Considerando estas cuestiones, resulta relevante generar espacios dentro de la formación ingenieril que permitan al alumnado acercarse a herramientas tecnológicas que serán utilizadas en su quehacer profesional diario. En línea con ello y con iniciativas a nivel mundial, la programación informática aparece como un campo de estudio imprescindible en la formación de los futuros ingenieros, incluso desde los primeros años de estudio.

En consecuencia, se diseñó una experiencia piloto en el marco de la cátedra "Introducción a la Ingeniería Mecánica" (primer año - primer cuatrimestre) [5], con el objetivo de introducir al alumnado en la resolución de problemas abiertos de ingeniería desarrollando una simulación de un sistema mecánico sencillo, para la cual los alumnos debieran incorporar de manera autónoma conceptos básicos de programación. En este sentido, se propuso como consigna para el trabajo final resolver el modelado y simulación de una pelota rebotando sobre una superficie plana.

A partir de esta experiencia, en este artículo se presenta un análisis en profundidad de distintos aspectos claves, a partir de un estudio de caso exploratorio. Con esto se pretende establecer algunos lineamientos que puedan servir para diseñar futuras propuestas curriculares en las carreras de ingeniería desde perspectivas pedagógicas que, desde la modalidad de taller, integren conceptos de programación y se encuentren centradas en la exploración y el aprendizaje por resolución de problemas.

A continuación se presentan trabajos relacionados, se describe la experiencia desarrollada y la metodología empleada, para luego presentar los resultados y 
las conclusiones derivadas del estudio, aportando algunos posibles lineamentos para trabajos futuros.

\section{MARCO GENERAL DE LA PROPUESTA}

El crecimiento de las industrias, la necesidad de nuevos servicios asociados, y el fortalecimiento de la vinculación entre empresas y universidades, hacen que las ingenierías sean esenciales para consolidar el desarrollo industrial y potenciar el conocimiento para la innovación productiva en los países de Latinoamérica. En este sentido, los gobiernos nacionales han generado en la última década políticas específicas. Ejemplos de ello son el programa "El ingeniero del 2020" [6] en México, en Chile el "Plan Estratégico-Nueva Ingeniería para el 2030" [7] o en Colombia el Proyecto "El Ingeniero Colombiano del 2020" [8], entre otros. Todos estos proyectos y programas tienen como finalidad última el fomento de vocaciones para el estudio de las carreras científico tecnológicas, en particular la ingeniería, y la mejora integral de las propuestas académicas de grado en las diversas universidades de cada país. Como impacto, plantean el crecimiento del número de graduados para el desarrollo industrial y de empresas vinculadas a la producción de bienes y de servicios necesarios para el desarrollo de las economías locales y regionales.

Por su parte, Argentina propuso en el año 2010 el "Plan Estratégico para la Formación de Ingenieros" (PEFI), impulsado por la Secretaría de Políticas Universitarias, que tiene como finalidad incrementar la cantidad de graduados en ingeniería en un $50 \%$ en 2016, y en un 100\% en 2021; para asegurar en cantidad y calidad los recursos humanos necesarios, con el fin de hacer de Argentina un país desarrollado. Para ello, este plan trabaja en tres grandes ejes estratégicos: Proyecto para la mejora de indicadores académicos, Aporte de la universidad al desarrollo territorial sostenible, e Internacionalización de la ingeniería argentina [9]. En el marco del primero de los ejes citados, se desarrolló la reforma curricular que dio como resultado el Nuevo Diseño Curricular 2014 de la Carrera de Ingeniería Mecánica de la Facultad de Ciencias Exactas, Ingeniería y Agrimensura de la Universidad Nacional de Rosario. El mismo prevé una carrera de cinco años de duración distribuida en diez cuatrimestres con una carga horaria total de 4040 horas. El diseño curricular propone una organización básica en actividades curriculares, las cuales facilitan la organización de contenidos afines, teniendo en cuenta los contenidos básicos, la carga horaria, las construcciones metodológicas y las formas de evaluación y acreditación que se consideran beneficiosas para la apropiación de los saberes y capacidades previstos. Cada actividad curricular es una unidad que conforma en sí misma un proyecto pedagógico dentro del plan, con relativa autonomía, aunque sólo adquiere significación dentro de la totalidad, a través de una adecuada articulación [10].

En este marco, la actividad curricular "Introducción a la Ingeniería Mecánica" optó por proponer una modalidad pedagógica de Taller [11-12] en la cual se promueve la resolución práctica de situaciones de valor para la formación ingenieril. Esta modalidad es altamente formativa por cuanto apunta a la resolución práctica de problemas, promoviendo la apropiación de formas participativas y socializadas de asumir las prácticas, forma en la que habitualmente se desarrolla el ejercicio profesional. El desarrollo de las capacidades que involucran desempeños prácticos envuelve una diversidad y complementariedad de atributos. Esto se debe a que las situaciones prácticas no se reducen a un simple hacer, sino que se constituyen como un hacer creativo y reflexivo en el que se ponen en juego tanto los marcos conceptuales disponibles, como la búsqueda de otros nuevos que resulten necesarios para orientar, resolver o interpretar los desafíos de la producción y de la práctica. En este sentido, el Taller, como modalidad pedagógica, apunta al desarrollo de alternativas de acción, a la toma de decisiones y a la producción de soluciones e innovaciones para encararlos. De esta forma, se propone como instancia de experimentación para el trabajo en equipo, estimulando la capacidad de intercambio, la búsqueda de soluciones originales y la autonomía del grupo.

Considerando que los cambios introducidos en el plan de estudio son recientes y que la modalidad de taller puede adquirir diferentes particularidades según la asignatura en la que se desarrolle, resulta necesario estudiar las acciones formativas desarrolladas, aportando datos concretos que reporten la concreción de las modificaciones introducidas. Asimismo y considerando los lineamientos introducidos por las políticas educativas, es necesario reconocer y estudiar cómo dentro de estas acciones se presentan e introducen las TIC. 


\section{LA PROGRAMACIÓN EN EL HACER DEL INGENIERO}

La educación superior, así como otros niveles educativos, se encuentra constantemente atravesada por las innovaciones. En este sentido, diferentes recursos tecnológicos se utilizan históricamente en la enseñanza de la ingeniería [13-14-15], dentro de los cuales se destacan la programación por computadora [16-17-18]. Sin embargo, aunque existen diferentes hardware y software que proveen a los docentes y estudiantes de herramientas sofisticadas para programar, el uso de estas herramientas de manera uniforme afectando en profundidad la forma en que se enseñan los contenidos, continúa siendo un desafío [19].

Por otra parte, crear código es una tarea compleja que implica la comprensión de conceptos ya no solo de ingeniería, sino también de programación informática. La mayoría de los alumnos que ingresan a la universidad carecen de los contenidos previos necesarios para abordar esta tarea [20], y deben aprenderlos en un período corto de tiempo [21], por lo que esta área suele ser un terreno complicado para aquellos que comienzan su camino por el mundo universitario. A esto se suma que no todos los estudiantes que ingresan a carreras de ingeniería cuentan con estudios secundarios técnicos.

Para hacer frente a esta situación, se han desarrollado múltiples experiencias que buscan introducir al alumnado ingresante en el mundo de la programación. En este sentido, las propuestas pedagógicas en el ámbito universitario apuntan a introducir esta temática utilizando diferentes recursos tecnológicos, como plataformas de Arduino [22], robots [23] o entornos como E-Toys, o Scratch, entre otros. Frente a la diversidad de herramientas disponibles, la revisión de experiencias realizadas en educación superior permite identificar cierta tendencia favorable hacia el uso de Scratch, desarrolladas desde el año 2003 por el grupo "Lifelong Kindergarten" perteneciente al MIT (Instituto Tecnológico de Massachusetts) [24]. Esta herramienta propone un lenguaje de programación basado en bloques agrupados en categorías que son utilizados para manipular los atributos de los objetos. Aunque originalmente fue creado para un público más joven, diferentes estudios señalan su potencial en el ámbito universitario, particularmente en propuestas de enseñanza de programación para alumnos ingresantes. Las investigaciones señalan que el hecho de que Scratch trabaje con el encastre de bloques para armar los programas, permite que los estudiantes que se inician en la programación puedan concentrarse en la resolución del problema, incorporando la sintaxis en tanto arman los algoritmos [25], centrándose primero en los problemas lógicos [26]. Asimismo, los estudios relevados coinciden en señalar esta herramienta como una puerta de entrada a lenguajes más complejos como Java, C [27] o Pascal [28], destacando además el potencial que tiene Scratch para motivar a los estudiantes, permitiendo el acceso a nociones fundamentales de programación. En relación con esto, otro estudio señala que pueden trabajarse los conceptos de variable, iteración, sincronización, listas y objetos [20]. En este sentido, vale señalar que aunque existen otras herramientas utilizadas con programadores novatos que también permiten un acercamiento a lenguajes más avanzados (por ejemplo Alice, Karel the robot, etc.), los estudios en educación superior señalan la flexibilidad que ofrece Scratch para construir múltiples tipos de proyectos, así como su curva de aprendizaje no tan pronunciada como en otros casos [26]. También se destaca la existencia de una extensa comunidad online, material de ayuda disponible (como wikis y tutoriales) y la posibilidad de observar por dentro los millones de proyectos disponibles en la versión online, que pueden ser tomados como referencia para realizar proyectos nuevos [24].

Por último, es importante destacar que esta temática ha sido ampliamente estudiada en el contexto europeo y anglosajón, pero no a en el contexto latinoamericano, donde la revisión de la literatura revela en su mayoría estudios aislados presentados en diferentes conferencias, sin ofrecer un análisis pormenorizado y en mayor profundidad que permita comenzar a sistematizar prácticas contextualizadas de talleres con TIC que puedan reflejar las particularidades de la región.

\section{MATERIALES Y MÉTODOS}

En este trabajo se presenta un estudio de caso exploratorio [29] realizado en relación al trabajo final integrador desarrollado en el marco de la actividad curricular "Introducción a la Ingeniería Mecánica”, durante el primer cuatrimestre de 2016. 
Particularmente en esta experiencia se decidió trabajar con Scratch, en su versión 2.0, por diferentes razones: 1) esta herramienta se encontraba disponible de manera gratuita y estable, lo cual facilita la utilización en universidades públicas argentinas, 2) en la plataforma online de Scratch se recogen alrededor de 8 millones de proyectos, que sirven como marco de referencia para el accionar de alumnos ingresantes, y múltiples proyectos relacionados con el problema planteado, 3 ) existen múltiples espacios de consulta y materiales disponibles que asisten el proceso de apropiación de la herramienta, lo cual favorece el autoaprendizaje, 4) ofrece un entorno visual amigable, sencillo de comprender e intuitivo, que facilita la apropiación de la herramienta por parte de los estudiantes.

En cuanto a los aspectos metodológicos, se ha optado por un estudio exploratorio, debido como se mencionaba previamente a la escasez de estudios relacionados. Se propone una metodología de trabajo mixta, en concordancia con lo propuesto por investigaciones previas en el área [30], que señalan la necesidad de un abordaje integral.

Los participantes del taller han sido el profesor titular de la cátedra, una investigadora y los alumnos de la asignatura de la comisión turno noche, que se organizaron en pequeños grupos.

En cuanto a los datos recogidos durante la experiencia, pueden señalarse diferentes recursos. Como instrumentos cuantitativos se utilizaron 3 encuestas elaboradas ad hoc: una general que relevaba formación previa y otras cuestiones que permiten caracterizar el grupo de estudiantes, y dos propias del proyecto con Scratch, al comienzo de la actividad y al cierre. Asimismo, se recopilaron los informes finales de cada grupo que siguen un protocolo específico de redacción planteado por la cátedra al comienzo de la actividad, permitiendo plasmar el proceso constructivo del modelo y de la simulación en su totalidad. Dicho protocolo recoge tanto la definición del problema como una copia del código final elaborado en Scratch, solicitando a su vez la explicitación de dificultades encontradas a lo largo del trabajo y su resolución por parte del grupo. También se relevaron las simulaciones realizadas en Scratch, recogidas en un estudio creado a tal fin. ${ }^{3}$

\footnotetext{
3 Ver: https://scratch.mit.edu/studios/1976294/
}

Por otra parte, los encuentros presenciales se realizaron durante el horario de la cátedra, y fueron grabados en audio. El análisis de los datos recogidos se realizó con la ayuda de software específico. Las encuestas fueron procesadas con SPSS 20 [31] y los audios, informes y proyectos con Atlas Ti 7.0 [32].

\section{RESULTADOS Y DISCUSIÓN}

En este apartado, organizado en tres secciones diferenciadas se presentan y discuten los principales resultados. Primeramente se expone el análisis del taller en sus características claves así como el perfil de los participantes. Seguidamente, se destacan las diferentes aproximaciones al problema, particularmente desde los espacios de consulta y las estrategias de colaboración desplegadas. Por último, se señalan problemáticas identificadas por los grupos durante la construcción de la simulación del sistema mecánico con la herramienta de programación propuesta.

\section{Propuesta del taller y participantes}

Para comprender los posibles alcances del proyecto realizado, es importante señalar algunas características particulares del contexto en el que se ha llevado a cabo, en este caso la asignatura "Introducción a la Ingeniería Mecánica”. Esta actividad curricular plantea perspectivas pedagógicas activas, desde una modalidad de taller físico-virtual [33], abordando la práctica analítica sobre casos de la Ingeniería Mecánica, en el marco interactivo y comunicacional que brindan las TIC, como apoyo a los procesos de enseñanza y de aprendizaje. En este sentido, la práctica participante en las diversas actividades propuestas en función de los objetivos planteados, es constitutiva al propio contenido de la formación que se fundamenta, adoptando una dinámica constructivista dialéctica.

En este marco, los desarrollos teóricos y prácticos se trabajan interrelacionando contenidos, promoviendo el aprendizaje colaborativo y la producción cooperativa. De esta forma, la reflexión y discusión de los distintos temas se construye a partir de exploraciones o producciones analíticas realizadas en pequeños grupos. Con este enfoque, se proponen una totalidad de diez trabajos a lo largo del cuatrimestre. La mitad de ellos son individuales y la otra mitad grupales. El último de los trabajos busca tener un carácter integrador de las diversas temáticas y ser 
propositivo en cuanto a las habilidades y actitudes señaladas como necesarias en la labor del ingeniero. La experiencia que aquí se analiza se desarrolló en el marco de este último trabajo, que en este caso constituyo el proceso de diseño, construcción y validación de una simulación.

En cuanto al sistema mecánico propuesto para simular, cabe mencionar que se optó por este ejemplo por tres razones: los conceptos necesarios para la construcción de la simulación son desarrollados en la asignatura "Introducción a la Física", que se dicta en paralelo. De esta forma, se fortalece la articulación entre actividades curriculares [34]. En segundo lugar, se contempló la facilidad en la realización experimental de la actividad: bajo costo y facilidad en la obtención de los materiales necesarios. Por último y no menos importante, este problema es retomado en tercer año en la asignatura "Dinámica de los Sistemas Físicos" con una complejidad mucho mayor, utilizando otro lenguaje de programación y simulación. De esta forma, se pretende generar una articulación longitudinal de la carrera, que plantea una visión espiralada respecto a la resolución de los problemas de ingeniería, logrando mejoras en las soluciones a partir de la profundidad técnica.

La experiencia que se presenta comprendió seis encuentros presenciales de una hora y media cada uno, desarrollados en el laboratorio de informática. En su diseño se hizo hincapié en la exploración y el trabajo autónomo por parte de los grupos. A partir de la reconstrucción de la experiencia, se identifican 3 momentos claves, como se observa en la Tabla 1 .

Como se observa en la tabla, siguiendo los lineamientos de la modalidad de taller, se privilegió a lo largo de los encuentros los espacios de discusión y puesta en común, que permitieran dialogar sobre los problemas identificados, las resoluciones implementadas y la aproximación a la herramienta. Asimismo, se destacan las situaciones prácticas, tanto en la realización del video experimental como en la construcción de la simulación.

Por otra parte, se trabajó con los estudiantes de la comisión de la noche, conformada inicialmente por 19 estudiantes. Sin embargo, debido al desgranamiento propio del primer año, participaron de la experiencia de manera completa 15 alumnos, edad media $=19,33$. El 40\% de los estudiantes
Tabla 1. Resumen de actividades realizadas.

\begin{tabular}{|c|c|}
\hline $\begin{array}{l}\text { Introducción } \\
\text { a Scratch y } \\
\text { presentación } \\
\text { del problema } \\
\text { (Encuentro 1) }\end{array}$ & $\begin{array}{l}\text { - Presentación del sistema } \\
\text { mecánico - Presentación de } \\
\text { Scratch (entorno, tipos de } \\
\text { bloques, proyectos online, } \\
\text { web, wiki, foros). } \\
\text { - Creación de cuentas de } \\
\text { usuario en Scratch. } \\
\text { - Realización de tutoriales que } \\
\text { aparecen en la web de Scratch. } \\
\text { - Consigna grupal: realización } \\
\text { de un video donde se observa } \\
\text { una pelota caer (video } \\
\text { experimental). }\end{array}$ \\
\hline $\begin{array}{l}\text { Experimentación } \\
\text { guiada } \\
\text { (Encuentros 2-4) }\end{array}$ & 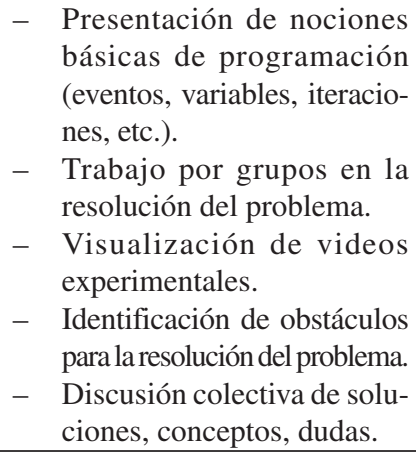 \\
\hline $\begin{array}{l}\text { Preparación y } \\
\text { presentación de } \\
\text { simulaciones } \\
\text { e informe } \\
\text { (Encuentros } 5 \\
\text { y 6) }\end{array}$ & $\begin{array}{ll}\text { - } & \text { Puesta en común de avances, } \\
\text { dudas, tareas pendientes. } \\
\text { - } & \text { Resolución de problemas. } \\
- & \text { Validación comparativa de los } \\
& \text { resultados de la simulación } \\
& \text { con el video experimental. } \\
- & \text { Presentación de proyectos e } \\
& \text { informes. } \\
- & \text { Discusión y debate sobre las } \\
\text { diferentes resoluciones. }\end{array}$ \\
\hline
\end{tabular}

contaba con formación secundaria técnica, mientras que el $86,7 \%$ desconocía Scratch. En cuanto a conocimientos previos de programación, el 53,3\% no había programado previamente. Los estudiantes se agruparon aleatoriamente estableciendo seis grupos de entre 2 y 4 participantes.

Aproximación al problema en el marco del taller: espacios de consulta y colaboración

Como se mencionó previamente, la experiencia se enmarcó en el formato taller, desde donde se buscaba el desarrollo de alternativas de acción, la toma de decisiones y la producción de soluciones e innovaciones en la resolución de problemas. En relación con ello, interesaba conocer cómo los estudiantes se aproximaban al problema planteado 
(la construcción del modelo y de la simulación) en un contexto de experimentación que fomentaba el trabajo en equipo. Para responder este interrogante se elaboró una encuesta que indagara sobre algunos aspectos de este proceso.

Primeramente, se les consultó a través de la encuesta inicial por su accionar si tenían una duda en cuanto a cómo resolver un problema. Un 73,3\% señaló como fuente a amigos/conocidos o familiares, mientras que el $26,7 \%$ restante recurre a buscadores web. Es importante señalar que esta pregunta contaba con otras dos opciones: libros y foros especializados, que no fueron escogidas. Estos datos iniciales contrastan con los comentarios registrados en audio durante la presentación de sus trabajos y lo señalado en sus informes. En estos datos se observa que los estudiantes consultaron diferentes fuentes online, tanto sobre el funcionamiento de Scratch como sobre las fórmulas que necesitaban para realizar la simulación. Cada grupo recurrió a diferentes recursos virtuales, como se observa en la Figura 1:

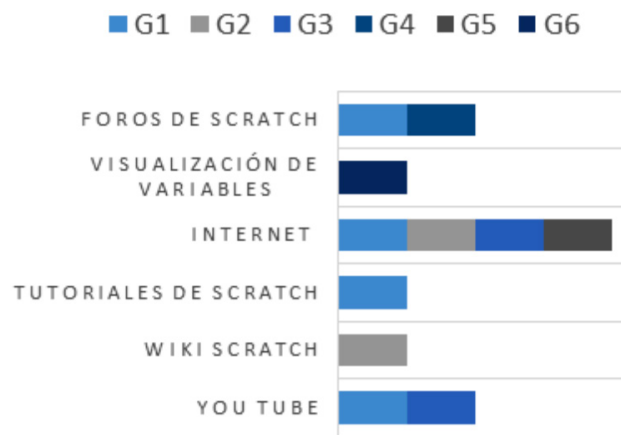

Figura 1. Fuentes de consulta identificadas.

Fuente: Elaboración propia.

Algunos grupos recurrieron a múltiples espacios de consulta mientras que otros solo utilizaron uno, como en el caso de grupo 5, que consultó solo en foros. Algunos de los grupos realizaron búsquedas más generales y no especifican puntualmente qué tipo de recursos emplearon para solucionar problemas o evacuar dudas. Por el contrario, otros grupos detallaron recursos más específicos, retomando incluso aquellos que se presentaron en los primeros encuentros presenciales, como la wiki, foros y tutoriales propios de Scratch. Vale mencionar que al consultarles si habían utilizado la ayuda propia de la herramienta, que aparece en una esquina de la interfaz bajo un signo de pregunta, todos los grupos respondieron negativamente.

Por otra parte, la encuesta inicial también indagó sobre cómo les resultaba más sencillo resolver un problema: un 46,7\% respondió con un compañero, un $40 \%$ en forma individual, y un $13,3 \%$ en forma grupal.

El análisis de los informes y los audios brindó nueva información en este punto, ya que 5 de los 6 grupos destacaron en la conclusión del informe que la actividad había fomentado el trabajo en equipo destacando además cuestiones como:

- Importancia del trabajo en equipo para el ejercicio profesional y la formación como ingenieros.

- Mejora de la productividad y capacidad organizativa, al identificar roles de los participantes y tareas a realizar.

- Identificación de fortalezas y debilidades de cada componente y su aporte al grupo como totalidad.

- Puesta en juego de procesos de toma de decisiones.

- Reconocimiento y consenso en cuanto a las diferencias en opiniones, conocimientos, propuestas.

En palabras de los participantes:

Grupo 4: "destacamos el fomento del trabajo en equipo; entendiendo que todas las partes componentes del grupo cumplen un rol específico, y sin la cual la tarea no hubiera podido ser llevada a cabo con éxito y, sin dejar de mencionar, el nivel de organización que debimos alcanzar para avanzar en la realización del proyecto" (Informe final).

Grupo 5: "Esta experiencia nos ayudó a desarrollar el trabajo en grupo que en un principio nos costó. Además desarrollamos un pensamiento más estructurado, que nos permitió adaptarnos a las limitaciones de Scratch" (Informe final).

Grupo 6: " también nos sirvió para trabajar en equipo, para que entre los integrantes del grupo demos nuestra opinión y podamos llegar 
a algo concreto mediante la fusión de las ideas individuales" (Informe final).

A partir de estos datos se desprende por un lado, un posible cambio de perspectiva en cuanto al trabajo en forma grupal, que aparece preponderado luego de la experiencia de trabajo conjunta en el taller en torno a una herramienta de programación con bloques.

Por otro, se observa una diversificación en cuanto a los recursos que se emplearon para resolver el problema de la simulación, recurriendo a múltiples fuentes, de entre las que se destacan los recursos en línea.

\section{Construyendo la simulación: problemáticas identificadas}

Tal como se señaló previamente, la herramienta propuesta en esta experiencia ha sido señalada como idónea para introducir a una gran diversidad de personas al mundo de la programación. Sin embargo, aunque los grupos en su mayoría lograron construir la simulación y resolver la actividad propuesta, la misma no estuvo exenta de obstáculos, como en cualquier proceso de resolución de problemas.

En este sentido, se indagó acerca de los problemas u obstáculos que encontraron los participantes de la experiencia al utilizar Scratch, a través de una pregunta abierta dentro de la encuesta administrada al cierre del taller. Como se observa en la Figura 2, el análisis de las respuestas permitió identificar diferentes problemáticas que también se reiteraron en los informes y registros de audio.

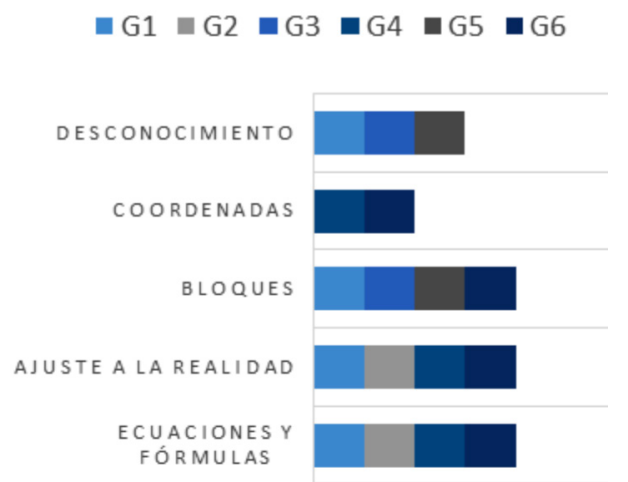

Figura 2. Problemáticas identificadas. Fuente: Elaboración propia.
Los problemas más señalados se relacionan con la programación por bloques, el ajuste a la realidad y las ecuaciones y fórmulas. Asimismo, los problemas destacados son mencionados por los mismos 4 grupos, lo cual revela una conexión entre las problemáticas, que se observa en el propio discurso de los estudiantes:

Grupo 2: "Se buscó la manera de lograr insertar nuestra fórmula con sus respectivos resultados dentro del programa ya que con la ecuación íbamos a lograr que la simulación se pareciera lo más posible a la realidad, este paso costó bastante ya que no lográbamos entender cómo introducir la ecuación por medio de los bloques que presenta el programa" (Informe final).

Grupo 3: "Otro problema fue que notamos que dependiendo el tiempo que pasaba entre darle comienzo al programa entre una simulación y otra variaba la velocidad de la pelota lo que no condice con la realidad" (Informe final).

Grupo 5: "Hay que tener en cuenta que la simulación en Scratch no es exacta ya que no tuvimos en cuenta el rozamiento del aire, imperfecciones de la pelota, coeficiente de rebote, etc. para poder simplificar el problema" (Informe final).

Grupo 7: "Para la mayoría del grupo no era la primera vez que veíamos la ecuación de la caída libre, lo difícil fue llevarla al programa, que también el modelo no responde un $100 \%$ a la realidad de lo que pasa con la pelota" (Audio. Presentación final).

Asimismo, esta relación entre problemáticas se hace visible en todos los grupos, por ejemplo, en el grupo 6, donde se combina el desconocimiento con la comprensión del funcionamiento de las variables y de la programación por bloques:

Grupo 6: "Muchos de los bloques tenían funciones que desconocíamos y en ocasiones las funciones de unos podían ser parecidas a las de otros pero con pequeñas diferencias que complicaban el funcionamiento del modelo. Como ejemplo proponemos el caso de los bloque-fijar variable $a-y$-cambiar variable por-". (Informe final). 
Por otra parte, algunos de los problemas se presentaron de manera más puntual en ciertos grupos, como la comprensión del sistema de coordenadas. En relación con esto último, vale aclarar que Scratch utiliza los ejes X-Y estableciendo el punto $(0,0)$ en el centro de la pantalla. En relación con ello, los participantes señalaron:

Grupo 7: "Lo tratamos de hacer de varias maneras, por ejemplo, un inconveniente era que el eje de referencia está en el medio, entonces tratamos de utilizar la fórmula de la posición, pero llegaba un momento que se hacía negativa la posición, nos cambiaba el signo y hacia disparar el valor y nos quedaba mal, entonces una solución fue eliminar la posición de la ecuación y nos quedamos con dos variables: tiempo y velocidad". (Audio. Presentación final).

Grupo 5: - "El problema que le veo yo al Scratch es que es bastante impreciso en las coordenadas, porque a la pelota yo la tiré de $145 \mathrm{~cm}$ hasta el piso, y yo le puedo poner la altura en 145 pero tengo que hacer que rebote o en la paleta o que rebote en el suelo. Si yo le pongo 145 y le pongo que rebote en la paleta no rebota cuando toca la paleta en el 0 digamos, como que se superpone un poco, la máscara de colisión no coincide con la imagen" (Audio. Presentación final).

Cabe señalar que varios estudiantes marcaron como dificultad el propio desconocimiento de la herramienta, y en algunos casos, de nociones de programación en general o de software específico, lo que condice con la propia estructura de la muestra en cuanto a saberes previos, como se destacó previamente. En relación con esto, los datos muestran diferentes opiniones respecto a la programación con bloques, dependiendo a su vez de los conocimientos previos de los estudiantes, como se observa en sus discursos:

Grupo 6: "Si bien conocíamos las fórmulas y ecuaciones físicas que necesitábamos utilizar en el modelo, no sabíamos cómo aplicarlas al mismo mediante los bloques" (Informe final)

Grupo 2: "al darte todo en bloques, ya todo predeterminado por el programa, no tenés la oportunidad o la opción de poner vos tu manera por ejemplo, de las formulas, entonces como que estás limitado con lo que te da el programa" (Audio. Presentación final).

Grupo 4: "Básicamente es lenguaje de programación que nosotros no conocemos y eso viene todo en un solo bloque lo cual facilita muchísimo, sino hubiéramos tenido que ponernos a escribir el código, es mucho más fácil que cualquier otra cosa" (Audio. Presentación final).

Grupo 7: "Para mí personalmente fue bastante complicado porque dentro de lo que estoy acostumbrado, el formato ese de bloques tiene un montón de limitaciones, en lo que yo uso tenés como una hoja en blanco en la que podes hacer lo que vos quieras, y acá estás limitado a un formato" (Audio. Presentación final).

Se observa que aquellos con mayores conocimientos encontraron restrictivo el formato de bloques, mientras que los que no contaban con conocimientos previos, agradecieron que la programación se realizara de esa manera.

En síntesis, los resultados presentados señalan las diferentes dificultades de los estudiantes durante el proceso de construcción de sus simulaciones. Pese a ello, 5 de los 6 grupos lograron realizar un prototipo y validarlo con el video experimental que realizaron al comienzo del taller. El grupo restante, realizó un prototipo, pero éste no pudo ser validado, ya que no logró el rebote esperado.

El relevamiento de las problemáticas y la identificación de su articulación develan la necesidad de comprender diferentes aspectos del entorno cuando se programa con el mismo. Estos obstáculos no impidieron, en líneas generales, la selección y utilización de los diferentes tipos de bloques que presenta la interfaz de Scratch, así como una adecuada organización de los mismos para poder responder a la consigna planteada.

\section{CONCLUSIONES}

A partir de los resultados, se observa cómo los participantes, que contaban con un bagaje diverso en cuento a nociones de programación y uso de 
entornos de este tipo, han podido apropiarse de la herramienta y construir sus simulaciones, alcanzando así el objetivo propuesto desde la asignatura en el marco de su trabajo final. En este sentido, es posible identificar algunas cuestiones que resultan claves como aportes de la presente experiencia a la formación ingenieril, particularmente en lo que atañe al uso de recursos informáticos dentro de la formación práctica desde el formato taller, un punto destacado por los planes de mejora de la calidad educativa, como revelaron los antecedentes.

Por un lado, el análisis de los espacios de consulta empleados por el alumnado, muestra que la participación en la experiencia ha fomentado la utilización de espacios virtuales, que al comenzar la actividad, no parecían mayormente utilizados. Recurrieron a diferentes páginas en internet, a recursos específicos e incluso a material en diferentes formatos, como por ejemplo en video. Sin embargo, en futuros trabajos, puede resultar útil destacar con mayor énfasis algunas alternativas de ayuda, como en el caso de Scratch, la incluida dentro de la propia interfaz, que pasó desapercibida para todos los grupos. Se destaca asimismo, la importancia de utilizar una herramienta con gran presencia en los entornos virtuales, ya que esto facilita la búsqueda de espacios de consulta por parte de los usuarios.

Por otro lado, al plantear un trabajo grupal como requisito necesario para enfrentar la consigna propuesta, los estudiantes pudieron identificar aspectos positivos respecto a estos espacios, dato que también difiere de sus apreciaciones al comenzar la asignatura. En este sentido, la experiencia realizada aporta al desarrollo de las habilidades sociales de los futuros ingenieros, fomentando el intercambio, la discusión y la reflexión, objetivos que también se planteaban desde el formato de taller propuesto, en línea con las políticas señaladas.

A su vez, como toda experiencia piloto, resulta imprescindible estudiar los problemas que puedan presentarse durante su desarrollo. En este sentido, el trabajar con la herramienta Scratch supuso ciertos desafíos, que deben ser considerados a la hora de diseñar actividades futuras. En cuanto a las problemáticas, algunas tienen directa relación con las características de la herramienta escogida en el estudio, aunque las mismas pudieron ser superadas una vez alcanzado cierto manejo de la misma. En este sentido, el presente estudio abona lo señalado en investigaciones previas respecto a la curva de aprendizaje de la herramienta, ya que los estudiantes pudieron alcanzar un alto grado de control en un breve lapso de tiempo.

En otro orden de cosas, cabe destacar las diferencias en cuanto a la valoración de la herramienta propuesta por parte de estudiantes con o sin conocimientos previos de programación. En este sentido, Scratch parece ser más apropiada para alumnos con escasos o nulos conocimientos previos en esta temática, que agradecen la presencia de bloques y de objetos, mientras que aquellos que manejan otros lenguajes de programación, consideran como limitación. Este dato avanza en lo planteado previamente por otros estudios, que destacaban el potencial de la herramienta para la introducción de nociones de programación, como puntapié inicial para comenzar a comprender la temática.

En síntesis, la experiencia piloto aporta evidencias en torno al potencial de la modalidad de taller para incluir TIC dentro de la formación práctica de futuros ingenieros, permitiendo el desarrollo de diferentes habilidades destacadas por las políticas universitarias vigentes. Así mismo, dentro de la gran variedad de TIC disponibles, la construcción de simulaciones con entornos de programación por bloques ha sido una elección acertada en cuanto ofrece a los estudiantes un primer contacto con la programación, colaborando en la generación de un piso tecnológico común entre aquellos con formación técnica y conocimientos previos y aquellos que no.

En esta línea, estudiar cómo pueden establecerse articulaciones entre asignaturas iniciales de la formación ingenieril con otras más específicas, como en este caso aquellas donde se estudian lenguajes de programación más complejos, aparece como una tarea pendiente para futuros estudios. Asimismo, analizar qué conceptos de programación pueden ser aprendidos mediante el uso de entornos con bloques abre posibles rumbos para nuevos estudios.

\section{REFERENCIAS}

[1] Facultad de Ciencias Exactas. "Ingeniería y Agrimensura". Fecha de consulta: 03/11/2016. URL: http://www.fceia.unr.edu.ar/ 
[2] Universidad Nacional de Rosario. Fecha de consulta: 03/11/2016. URL: http://www.unr. edu.ar/

[3] Resolución N ${ }^{\circ}$ 576-13. Comisión Nacional de Evaluación y Acreditación Universitaria (CONEAU-Ministerio de Educación de la República Argentina), Buenos Aires, 02 de agosto de 2013. Fecha de consulta: 03/11/2016. URL: http://www.coneau. gov.ar/archivos/resoluciones/Res57613E804076611.pdf

[4] Resolución de Acreditación N ${ }^{\circ}$ 57213. Comisión Nacional de Evaluación y Acreditación Universitaria (CONEAUMinisterio de Educación de la República Argentina). Acreditación de Calidad Académica MERCOSUR de Carreras Universitarias Sistema ARCU-SUR - Red de Agencias Nacionales de Acreditación (RANA). 29 de julio de 2013. Fecha de consulta: 03/11/2016. URL: http://www. coneau.gov.ar/archivos/resoluciones/Res57213E804019410.pdf

[5] G. Rodríguez y N. Monjelat. "Simulación De Sistemas Mecánicos Simples Con Scratch: Un Estudio De Caso En Introducción a La Ingeniería”. En A. Farias, J. Pilar y C. Acuña (Eds.), 3er Congreso Argentino de Ingeniería - CADI 2016 (pp. 39-49). Resistencia, Chaco: Universidad Tecnológica Nacional - Facultad Regional Resistencia. 2016.

[6] O. Rascón Chávez. "Panorama de la ingeniería en México". Academia de Ingeniería de México Editora. Distrito Federal, México. 2012.

[7] Plan Estratégico - Nueva Ingeniería para el 2030. Corporación de Fomento de la Producción - Gobierno de Chile. Fecha de consulta: 03/11/2016. URL: http://www. corfo.cl/programas-y-concursos/programas/ plan-estrategico--nueva-ingenieria-para-el2030--regiones

[8] V. Albéniz Laclaustra y L. González Araujo. "El ingeniero colombiano del año 2020. Retos para su formación". Asociación Colombiana de Facultades de Ingeniería - ACOFI. Gráficas Editores. Bogotá, Colombia. 2007.

[9] Plan Estratégico para la Formación de Ingenieros. Ministerio de Educación. República Argentina. Fecha de consulta: 03/11/2016. URL: http://pefi.siu.edu.ar/
[10] Plan de Estudios 2014 - Carrera Ingeniería Mecánica. Facultad de Ciencias Exactas, Ingeniería y Agrimensura, Universidad Nacional de Rosario. Fecha de consulta: 03/11/2016. URL: http://web.fceia.unr. edu.ar/images/PDF/carreras_de_grado/ Planes_2014/10102014/PLAN_2014_ MECANICA.pdf

[11] D. Braccialarghe, B. Introcaso y G. Rodríguez. "Hacia la construcción de la modalidad de taller como propuesta de integración entre introducción a la ingeniería y las ciencias básicas". Revista Argentina de Enseñanza de Ingeniería. Número 9, pp. 41-50. 2015.

[12] P. San Martín y G. Rodríguez. "Construir un nuevo diseño curricular participando de un Dispositivo Hipermedial Dinámico". Revista Cognición. Revista Científica de FLEAD. Número 22, pp. 2009.

[13] F.A. Kulacki y E.R. Krueger. "Trends in Engineering Education - An International Perspective". Proceedings of the World Conferences on Engineering Education Annual Meeting. Rio de Janeiro, Brazil. 1998.

[14] R. Pessacq, C. Paniagua y O. Iglesias. "Utilización de Herramientas Informáticas en la Enseñanza de la Ingeniería". II Workshop de Investigadores en Ciencias de la Computación. La Plata, Buenos Aires. 2000.

[15] D. Giulianelli, R. Rodríguez, P. Vera, A. Trigueros, E. Moreno y I. Marko. "Incorporación de Recursos Audiovisuales como Apoyo al Aprendizaje en carreras de Ingeniería". Workshop de Tecnología Informática Aplicada en Educación. Buenos Aires, Argentina. 2014.

[16] A.J. Magana, S. P. Brophy y G.M. Bodner. 'Instructors' intended learning outcomes for using computational simulations as learning tools". Journal of Engineering Education, Vol. 101, N 2, pp. 220-243. 2012.

[17] S.P. Brophy, A.J. Magana y A. Strachan. "Lectures and simulation laboratories to improve learners' conceptual understanding". Advances in Engineering Education, Vol. 3, $\mathrm{N}^{\circ}$ 3, pp. 1-28. 2013.

[18] D. Fraser, R. Pillay, L. Tjatindi y J. Case. "Enhancing the learning of fluid mechanics using computer simulations". Journal of 
Engineering Education, Vol. 96, $\mathrm{N}^{\mathrm{o}} 4$, pp. 381-388. 2007.

[19] L.S. Fletcher. "Mechanical Engineering Education for the 21st Century". International Journal of Engineering Education, Vol. 13, $\mathrm{N}^{\circ}$ 1, pp. 42-51. 1997.

[20] P. Vera, E. Moreno, R. Rodríguez, C. Vázquez, F. Valles y M. Cornejo. "Plataforma de Gamificación para la Enseñanza de Programación en el Ámbito Universitario". XVIII Workshop de Investigadores en Ciencias de la Computación, pp. 905-909. Entre Ríos, Argentina. 2016.

[21] M. Rizvi, T. Humphries, D. Major, M. Jones y H. Lauzun. "A CSO course using Scratch". Journal of Computing Sciences in College, Vol. 26, $\mathrm{N}^{\circ}$ 3, pp. 19-27. 2011.

[22] R. Coppo, A. Cavallo y G. Ursua. "Sistema didáctico para la enseñanza de la programación con metodologías de aprendizaje basado en problemas". XIII Workshop de Investigadores en Ciencias de la Computación. Rosario, Argentina. 2011.

[23] T. Yamanishi, K. Sugihara, K. Ohkuma y K. Uosaki. "Programming instruction using a micro robot as a teaching tool". Computer Applications in Engineering Education, Vol. 23, No 1, pp. 109-116. 2015.

[24] J. Maloney, M. Resnick, N. Rusk, B. Silverman y E. Eastmond. "The Scratch Programming Language and Environment". ACM Transactions on Computing Education, Vol. 10, No 4, pp. 1-15. 2010.

[25] P. Willging, G.J. Astudillo y S.G, Bast. "El software de animación como una estrategia innovadora para el aprendizaje de lenguajes de programación". XIV Workshop de Investigadores en Ciencias de la Computación. Misiones, Argentina. 2012.
[26] U. Wolz, H.H. Leitner, D. Malan y J. Maloney. "Starting with scratch in CS 1". ACM SIGCSE Bulletin, Vol. 41, No 2. SIGSE 2009. New York, Estados Unidos. Marzo 2009.

[27] M. Resnick, J. Maloney, A. MonroyHernandez, N. Rusk, E. Eastmond, K. Brennan, A. Millner, E. Rosenbaum, J. Silver, B. Silverman y Y. Kafai. "Scratch: Programming for All". Communications of the ACM, Vol. 52, No 11, pp. 60-67. 2009.

[28] D.J. Malan y H.H. Leitner. "Scratch for Budding Computer Scientists". Proceedings of the Thirty-Eighth Sigcse Technical Symposium on Computer Science Education, pp. 223-227. Kentucky, USA. 2007.

[29] R.K. Yin. "Case Study Research: Design and Methods". SAGE. London. 2003.

[30] M. Borrego, E. Douglas y C. Amelink. "Quantitative, qualitative, and mixed research methods in engineering education". Journal of Engineering Education, Vol. 98, $\mathrm{N}^{\circ} 1$, pp. 53-66. 2009.

[31] IBM. "SPSS Statistics Base". Fecha de consulta: 03/11/20160. URL: http://www-03. ibm.com/software/products/es/spss-stats-base

[32] ATLAS.ti GmbH. "ATLAS.Ti. 7". Fecha de consulta: 03/11/2016. URL: http://atlasti. com/product/v7-windows/

[33] P. San Martín. "Aspectos sociales y tecnológicos del Dispositivo Hipermedial Dinámico desarrollados en diferentes contextos educativos". Revista de Educación, Año 4, No 5, pp. 81-98. 2013.

[34] V. Carbonell, C. Romero y M. Flórez. "Interactive simulations as teaching tools for engineering mechanics courses". European Journal of Physics. Vol. $34 \mathrm{~N}^{\circ}$ 4, pp. 9911004. 2013. 